\title{
Mean Stress Correction Based on Strength Degradation
}

\author{
Jingwei Cui ${ }^{a}$, Jinlong Liang ${ }^{b}$ \\ Shenyang University, Shenyang 110044, China

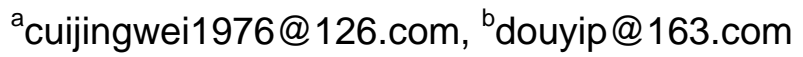

Keywords: strength degradation, mean stress correction, asymmetric cyclic stress.

\begin{abstract}
Life prediction of parts under complex loads has always been an important research topic in mechanical engineering design. In order to make the results more accurate, the stress correction formula for the asymmetric cyclic stress is derived mixed strength degradation law and the characteristics of 45 steel in this paper. The final results indicate that linear strength degradation model established in this paper can describe the strength degradation law of the fatigue process well.
\end{abstract}

\section{Introduction}

Along with the rapid development of engineering technology and materials science, especially in the aviation, aerospace, marine, transportation and other important industries, the failure mode of fatigue failure appears frequently. A research by U.S. Federal Government in 1982 show that accidents caused by fatigue fracture accounted for $95 \%$ of the total failure of mechanical structure. Fatigue life prediction of mechanical structures under complex loads has become an important research topic in the design of mechanical engineering at home and abroad.

Liyang Xie ${ }^{[1]}$ introduced the double parameter fatigue failure criterion which is closely related with residual strength and derived the formula of the mean stress correction under asymmetric cyclic loading from the Goodman formula by analyzing the function principle of the average stress.

And Liyang Xie ${ }^{[2]}$ pointed out traditional method of mean stress correction used in a constant amplitude fatigue test under non constant amplitude cyclic loading could not be used to calculate cumulative damage and traditional method need to be revised to eliminate static effect of mean stress.

Based on the results of finite element calculation, Jialei Guo ${ }^{[3]}$ calculated the fatigue damage of dangerous section under amplitude and equal amplitude asymmetric torsional stress loads through the life equivalent method and the damage equivalent method. The results showed damage equivalent method with mean stress effect could significantly improve the accuracy of fatigue life estimation in the calculation of fatigue life under complex loads.

An appropriate mean stress correction method is needed for different materials in life prediction of materials. In order to make the results more accurate, the stress correction formula for the asymmetric cyclic stress is derived mixed strength degradation law and the characteristics of 45 steel in this paper.

\section{Traditional Method.}

The method based on stress is the earliest method and the most common way in the life prediction. The stress $\mathrm{S}$ and fatigue life $\mathrm{N}$ are expressed in a pair of graphs called $\mathrm{S}-\mathrm{N}$ curve and the Basquin formula used to express the curve in the graph,

$S N^{m}=C$

In the equation, $\mathrm{m}$ and $\mathrm{C}$ are parameters related to the material, stress ratio, loading regime and other parameters, the formula also can be rewritten by

$S(2 N)^{m}=\sigma_{F}$

In the equation, $\sigma_{F}$ is tensile breaking stress, $\mathrm{m}$ is a parameter related to the material, stress ratio, loading regime and other parameters; 


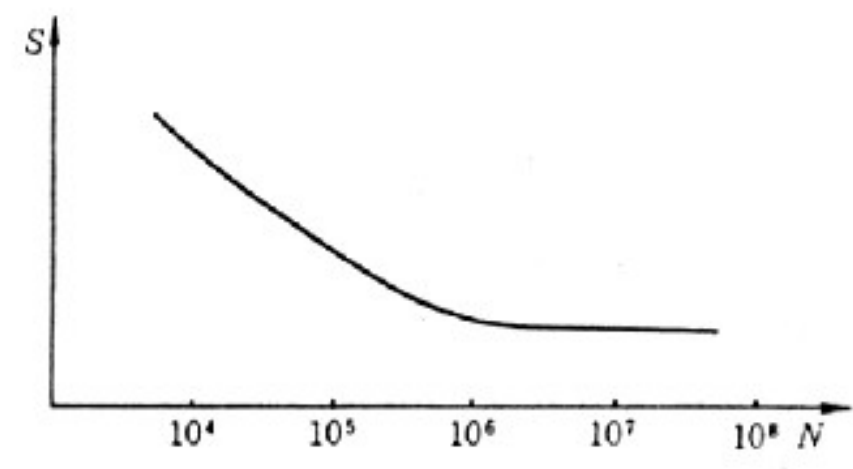

Fig. 1 S-N Curve

The equations above apply to fatigue life problem for symmetric cyclic loading. For the constant amplitude asymmetric load, the Basquin formula is modified,

$\sigma_{a}=\left(\sigma_{f}{ }^{\prime}-\sigma_{m}\right)\left(2 N_{f}\right)^{m}$

Fatigue life is

$$
N_{f}=0.5\left[\frac{\sigma_{a}}{\sigma_{f}{ }^{\prime}-\sigma_{m}}\right]^{\frac{1}{m}}
$$

\section{Mean stress correction formula}

\subsection{Strength Degradation Model.}

The mean stress effect is divided into static effect and dynamic effect. The static effect only affects the final fracture and dynamic effects on the evolution of damage in fatigue process and strength degradation rate. So the dynamic effect of average stress is considered in calculating cumulative damage. A force named damage equivalent stress for the analysis and calculation of complex loads is presented through the research results of traditional methods and analysis of some experimental data to modify the effect of mean stress on fatigue damage.

In this paper, a linear intensity degradation model is introduced to describe the degradation of residual strength.

$$
\sigma=\sigma_{0}-\frac{\left(\sigma_{0}-\sigma_{\max }\right) n}{N}
$$

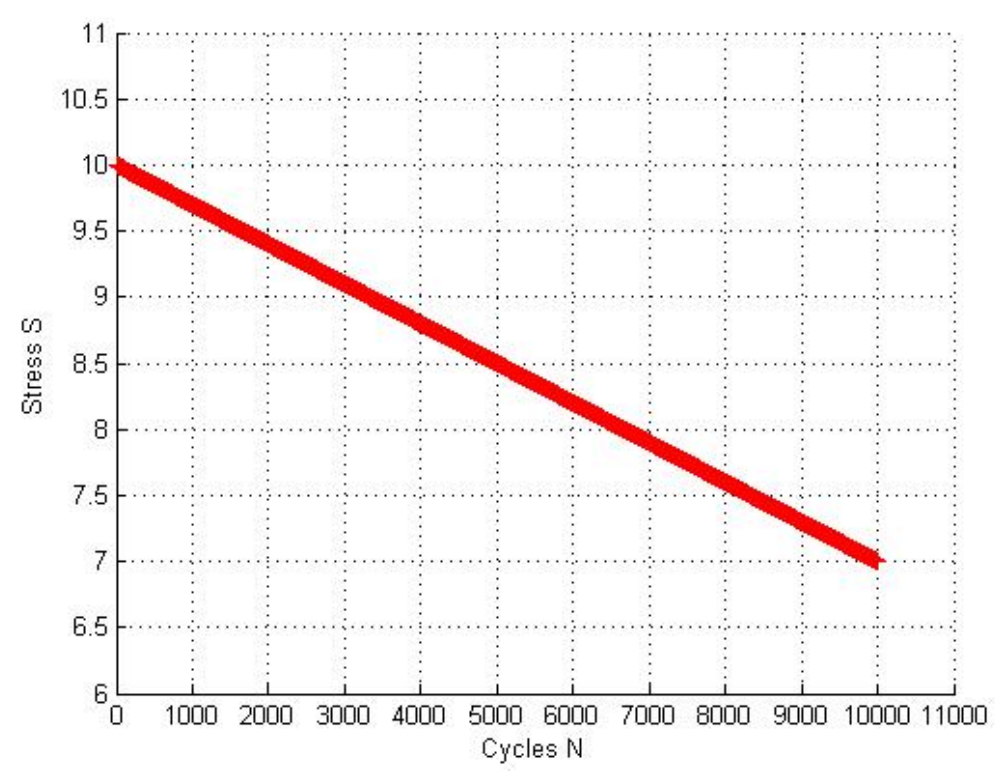

Fig. 2 Strength Degradation Curve 
Strength degradation rate can be expressed as,

$\frac{d \sigma}{d n}=\frac{\sigma_{\max }-\sigma_{0}}{N}$

When the load is constant amplitude symmetric cyclic load,

Cyclic stress with amplitude $\sigma_{a}$, mean $\sigma_{m}$ and life $N$ is equivalent to another symmetrical cycle with amplitude $\sigma_{d d}$ and life $N_{d d}$,

$$
\frac{\sigma_{0}-\left(\sigma_{\mathrm{a}}+\sigma_{m}\right)}{\sigma_{0}-\sigma_{d d}}=\frac{N}{N_{\mathrm{dd}}}
$$

\subsection{Modification of Normal Stresses.}

Most of the loads are asymmetric cyclic loads in practical applications, so it is still necessary to revise the mean stress. There are several kinds of average stress correction theory about the average tensile stress influence on fatigue strength.

1) Morrow relationship

$\sigma_{a}=\sigma_{-1}\left(1-\sigma_{m} / \sigma_{f}\right)$

2) Gerber parabola

$$
\sigma_{a}=\sigma_{-1}\left(1-\sigma_{m} / \sigma_{b}\right)^{2}
$$

3) Soderberg relationship

$\sigma_{a}=\sigma_{-1}\left(1-\sigma_{m} / \sigma_{s}\right)$

4) Goodman relationship

$\sigma_{a}=\sigma_{-1}\left(1-\sigma_{m} / \sigma_{b}\right)$

Goodman relationship is recommended to modify the average stress of the tensile strength of brittle materials including high strength steel which can be more accurate and the fatigue life is in good agreement with the experimental results; For most engineering alloys, Soderberg relationship is more conservative to estimate the fatigue life; For plastic material, it is more accurate to use the Gerber relationship to modify the mean stress to get the relationship of stress and fatigue life; Due to the wide applicability of the formula and the acceptable tolerance of life estimation, we choose the Soderberg relationship to revise the mean stress in this paper.

$$
\begin{aligned}
& \sigma_{d}=\sigma_{a}\left(1-\sigma_{m} / \sigma_{s}\right) \\
& \sigma_{s} \text { is the yield strength of material. } \\
& N=0.5\left[\frac{\sigma_{a}}{\sigma_{f}\left(1-\frac{\sigma_{m}}{\sigma_{s}}\right)}\right]^{\frac{1}{m}} \\
& \frac{N}{N_{d d}}=\left[\frac{\sigma_{a}}{\sigma_{d d}\left(1-\frac{\sigma_{m}}{\sigma_{s}}\right)}\right]^{\frac{1}{m}} \\
& \frac{\sigma_{0}-\left(\sigma_{\mathrm{a}}+\sigma_{m}\right)}{\sigma_{0}-\sigma_{d d}}=\left[\frac{\sigma_{a}}{\sigma_{d d}\left(1-\frac{\sigma_{m}}{\sigma_{s}}\right)}\right]^{\frac{1}{m}}
\end{aligned}
$$

Thus $\sigma_{d d}$ can be gotten, bring the data into the formula (13) and formula (13) the $N_{d d}$ can be gotten.

\section{Practical Calculation Examples}

In order to verify the rationality of the formula in this paper, 45 steel is chosen for calculation. The tensile strength $\sigma_{b}$ of steel is $600 \mathrm{MPa}$, yield strength $\sigma_{s}$ is $355 \mathrm{MPa}$. The value of the fatigue 
strength index $\mathrm{m}$ in the Basquin formula of the hard metal is -0.1 . The applied load amplitude $\sigma_{a}$ is $100 \mathrm{MPa}$, mean value $\sigma_{m}$ is $100 \mathrm{MPa}$.

Table 1 Chemical Composition of 45 Steel

\begin{tabular}{cccccc}
\hline & $\mathrm{C}$ & $\mathrm{Si}$ & $\mathrm{Mn}$ & $\mathrm{P}, \mathrm{S}$ & $\mathrm{Cr}, \mathrm{Ni}, \mathrm{Cu}$ \\
\hline Content(\%) & $0.42 \sim 0.50 \%$ & $0.17 \sim 0.37 \%$ & $0.50 \sim 0.80 \%$ & $\leq 0.035 \%$ & $\leq 0.25 \%$ \\
\hline
\end{tabular}

The fatigue life of the material is calculated by the traditional method is $9.8 \times 10^{8}$.

The damage equivalent stress $\sigma_{d d}$ of the strength degradation model with the modified mean stress is $131 \mathrm{MPa}$.

So equivalent fatigue life $N_{d d}$ is $1.6 \times 10^{8}$.

Table 2 Results of Mean Stress Correction

\begin{tabular}{ccc}
\hline & Equivalent Stress & Fatigue Life $N_{d d}$ \\
\hline Before Revision & - & $9.8 \times 10^{8}$ \\
After Revision & $131 \mathrm{MPa}$ & $1.6 \times 10^{8}$ \\
\hline
\end{tabular}

\section{Summary}

Appropriate calculation model must be chosen to predict life of different materials. By comparing with the traditional method of fatigue life prediction after correcting the strength degradation formula, the linear strength degradation model established in this paper can describe the strength degradation law of the fatigue process well and the final result meets the needs of the engineering application.

\section{References}

[1] Liyang Xie, Chen li, An Ping, Strength Degradation and Mean Stress Effect under Fatigue Loading, Journal of Mechanical Strength , Vol.18, No.3, p.41

[2] Liyang Xie, Wenqiang Lin, Hao Xu, et al. Mean Stress Amending Method for Fatigue under Complex Loading, Acta Aeronautica Et Astronautica Sinica, Vol.14, no.12, p.639

[3] Jialei Guo, Le Pang, Applicaton of Mean Stress Correction Method in Shafting Stress Calculation for Turbo-generator, Vol.41, No.2, p.68

[4] Chunhu Luo: Study on the Fatigue Properties of Shield Machine Fuse Shaft, (MS., Zhengzhou University, China 2012), p.14.

[5] Xie L Y, Wang Z, Xu H, Experimental Study on Cumulative Fatigue Damage Law under Two Stress Levels, Chinese J. Mech. Engng, Vol.1, No.1, p.90

[6] Henry D L, Theory of Fatigue Damage Accumulation in Steel, Asme trans, 1955, Vol.77, p.913 